\title{
Pattern Building Methods in Genetic Data Processing
}

\author{
Tatyana Volkova, Elena Furta, Olga Dmitrieva, Irina Shabalina
}

\begin{abstract}
This work is a part of long-term investigations carried out by the Laboratory of Molecular Genetics of Constitutive Immunity at Petrozavodsk State University. The paper describes development of approaches to genetic information analysis as applied to septic shock sensitivity investigation on basis of a mice model. The septic shock can be modeled by the injection of tumor necrosis factor (TNF). The paper describes two methods of finding markers combinations (patterns) affecting resistance to TNF. The developed algorithms were implemented and applied to the input data. The results showed the significant correspondence between some chromosome markers and resistance to TNF.
\end{abstract}

Index Terms-Genetic Analysis, Mathematical Methods of Diagnostics, Statistical Methods

\section{INTRODUCTION}

$\mathrm{D}$ IFFERENT species of animals have various levels of sensitivity to septic shock. A mice model is used for the investigation of human sensitivity. Previous investigations showed that septic shock can be modeled by the injection of the synthetically obtained tumor necrosis factor (TNF) [1], [2].

Two lines of mice were used in the investigation. The mice belonging to $B 6$ line have sensitivity to TNF similar to a human one, while the absolutely resistant to TNF mice belong to $M s m$ line.

The genetic analysis method can be applied to search the gene responsible for TNF sensitivity using two mice lines with opposite TNF sensitivity. This method considers the outcrossing of different lines of mice and the analysis of a phenotype and a genotype of the rising generation. The analysis is performed by comparison of the observed phenotype and the determined genotype for the rising generation objects.

Manuscript received May 15, 2014; accepted June 5, 2014. Date of online publication: June 30, 2014.

Authors are grateful for the Strategic Development Program of Petrozavodsk State University that provided required support for this research.

T. Volkova is with the Laboratory of Molecular Genetics of Constitutive Immunity at Petrozavodsk State University (e-mail: volkovato@yandex.ru).

E. Furta is with the Faculty of Mathematics at Petrozavodsk State University (e-mail: lena-furta@rambler.ru).

O. Dmitrieva is with the Faculty of Mathematics at Petrozavodsk State University (e-mail: dmitr_eva@mail.ru).

I. Shabalina is with the Chair of Applied Mathematics and Cybernetics, Faculty of Mathematics at Petrozavodsk State University (e-mail: i_shabalina@petrsu.ru).
There are two types of phenotypes: a resistant type (a mouse survives after TNF injection) and a sensitive type (a mouse dies after TNF injection).

The genotype can be represented by three types: "A" - a resistant genotype of line Msm, "B" - a sensible genotype of line $\mathrm{B} 6$ and " $\mathrm{H}$ " - a heterozygous genotype.

A genotype information unit is the marker, which describes information of the chromosome fragment where the genotype is detected. A sample of genotype and phenotype values was processed by mathematical algorithms.

The purpose of the investigation was to find the marker or the group of markers responsible for sensitivity or resistance to TNF. The search is based on the hypothesis that a phenotype corresponds to a genotype.

At first sight, the purpose could be accomplished with the help of some statistical criteria (for example, Pearson's phisquare). If only one group of genes on a chromosome (described by a marker) completely determined the sensitivity or resistance to $\mathrm{TNF}$ the problem would have a simple solution. However the previous investigations have not confirmed such assumption. Correlation between a genotype and sensitivity is a complex problem which cannot be solved by a single marker effect.

Another complication is that the problem statement does not agree with the "classical" one solved by the methods of correlation analysis, multiple regression analysis, ANOVA, pattern recognition [3, 4]. Typically approaches on singlemarker analysis and multi-marker analysis are based on chisquared test, likelihood-based test and logistic regression model $[5,6]$. At that a considerable amount of research has focused on selection of appropriate computational methods and software packages for geneticists and other biomedical challenges $[6,7]$. The graph approaches to present a human genome based on correlation and entropy are described in work [8]. Up to date statistical software [9] utilizes data mining algorithms with classification trees methods to provide hierarchic model of data. However, these algorithms are most suitable for the study of the predictor effect on a continuous result. In a case of large amount of predictors the methods create complex hierarchical structure which is hard to interpret and utilize.

Nevertheless, our work was focused on pattern building methods for modelling of the multiple discrete variables effect on a discrete result. These methods require the adaptation of standard methods and the development of particular methods and approaches.

This paper proposes several approaches to find markers combinations affecting sensitivity to TNF. They are based on 
correlation analysis, theory of probability and graph theory. The developed algorithms were implemented and applied to the input data.

\section{PROBLEM STATEMENT}

The initial data were collected by the Laboratory of Molecular Genetics of Constitutive Immunity at Petrozavodsk State University. The sample consisted of genetic information contained in 44 markers of 20 chromosomes. The marker values were detected for 213 mice: 137 with a sensitive phenotype and 76 with a resistant phenotype.

Let's present the initial sample $\Omega$ into classes: $\Omega_{R}$ containing resistant objects and $\Omega_{S}$ containing sensitive ones. It is obvious that $\Omega_{R} \cap \Omega_{S}=\emptyset$ and $\Omega_{R}+\Omega_{S}=\Omega$.

Let's describe the genetic data for object with number $j$ as a vector:

$x_{j}=\left(x_{m_{1}}^{1}, x_{m_{2}}^{1}, \ldots, x_{m_{n_{1}}}^{1}, x_{m_{1}}^{2}, x_{m_{2}}^{2}, \ldots\right.$

$$
\left.\ldots, x_{m_{n_{2}}}^{2}, \ldots, x_{m_{1}}^{20}, x_{m_{2}}^{20}, \ldots, x_{m_{n_{20}}}^{20}\right) \text {, }
$$

where $j=1,2, \ldots, N$ and $N$ - the sample volume, $x_{m_{k}}^{h}-$ genotype for marker on $h$-th chromosome with genetic distance equal $m_{k}$ centimorgan, $x_{m_{k}}^{h} \in\{A ; B ; H\}, h=$ $1,2, \ldots, 20, k=1,2, \ldots, n_{h}, n_{h}$ the number of markers on $h$-th chromosome, $\sum_{h=1}^{20} n_{h}=n$.

Let $\boldsymbol{X}(H, M, X)$ denote a pattern, which is a combination of several values of markers:

$\boldsymbol{X}(H, M, X)=\left(x_{m_{1}}^{h_{1}}=X_{1}, x_{m_{2}}^{h_{2}}=X_{2}, \ldots, x_{m_{n}}^{h_{n}}=X_{n}\right)$,

where $h_{k}$ - number of chromosome, $h_{k} \in H, m_{k}$ - genetic distance of marker, $m_{k} \in M, X_{k}$ - values of marker, $X_{k} \in X$, $k=1,2, \ldots, q$, value $q$ - the length of pattern. If the object $x$ has the combination of values (2), it means that «the object $x$ corresponds to the pattern $\boldsymbol{X}(H, M, X) »$.

As it was considered above, the problem is to find patterns with high connection with sensitivity or resistance to TNF.

\section{METHODOLOGY}

\section{A. Hierarchic patterns}

This method finds the patterns $\boldsymbol{X}(H, M, X)$ in accordance with some criteria of quality. The criteria can be: resistance probability maximization for the objects which correspond to the pattern:

$$
P(R(x)=1 \mid \boldsymbol{X}(H, M, X)) \rightarrow \max ,
$$

- belonging of such probability to given interval:

$$
P(R(x)=1 \mid \boldsymbol{X}(H, M, X))>P_{\text {opt }},
$$

where $P(\ldots \mid \ldots)$ - conditional probability, $R(x)$ - phenotype for object $x(0-$ sensitive, 1 - resistant $), P_{\text {opt }}$ - the value of probability. We should use enough high values of probability to assure the high survive probability for the objects connected with the pattern [10].

The preliminary stage of the method was required to define the markers with high connection with sensitivity or resistance to TNF. The rank correlations [11] were used to evaluate the degree of association between markers and resistance to TNF.

Rank variables $x_{m}^{h}=\{-1 ; 0 ; 1\}$ were used to describe initial genetic data for marker $m$ on chromosome $h$. The value " 1 " corresponds to genotype "A", "-1" corresponds to "B" and
" 0 " to "H". Rank variable $r=\{0 ; 1\}$, where " 1 " corresponds to resistant phenotype, " 0 " to sensitive one. As it was mentioned above the basic hypothesis of the investigation was "phenotype corresponds to genotype". So, the significant positive correlations match to the case when the resistant genotype connects with the resistant phenotype [12].

Patterns with hierarchic structure were built by the following method. The markers having significant positive correlations were put in the "root" of the hierarchic patterns. In the process the values " $\mathrm{A}$ " were put in the pattern root for $\Omega_{R}$ class, value "B" for $\Omega_{S}$ class.

The markers with high frequency of combination with the "root" marker were linked to the "root". If the probability of combination was more than $P_{o p t}$ the combination became the "pattern" consisting of two markers. The second marker in the combination presented the second level of the hierarchic pattern. At the next step the markers which had not been used were added to the previous level markers. The iteration process of markers addition should be continued until the probability of pattern meets the condition (4). When the process was completed we had a set of hierarchic patterns consisting of $2,3,4, \ldots, n$ levels. Such patterns had high association with the sensitivity or resistance to TNF.

\section{B. Patterns as a graph}

The method is based on frequency calculation of paired occurrence of marker values for each class of objects and on presentation the results as a graph [10].

At the first step we evaluated paired frequency $p_{i j}^{\alpha}, \alpha \in$ $\{S, R\}$ for each pair of marker values for each class of objects, $i, j=1,2, \ldots, 3 n$. The dimension $3 n$ connected with three concerned genotypes. The results were presented by two $3 n \times 3 n$ matrices $P_{S}$ and $P_{R}$ for $\Omega_{S}$ and $\Omega_{R}$ classes respectively.

Then we calculated differences $\Delta_{i j}^{S}=p_{i j}^{S}-p_{i j}^{R}$ for $\Omega_{\mathrm{S}}$ class and $\Delta_{i j}^{R}=p_{i j}^{R}-p_{i j}^{S}$ for $\Omega_{R}$ class, $i, j=1,2, \ldots, 3 n$.

The next step required selection of marker pair values $(i, j)$ for each class with differences larger than the prescribed parameter $0<\varepsilon<1$. These pairs were presented as the edges of graphs, the paired frequency $p_{i j}^{\alpha}, i, j=1,2, \ldots, 3 n, \alpha \in$ $\{S, R\}$ were defined as the weights of the edges.

The next edges could be added to the graphs by selection of the markers $k$ : such as $k=\operatorname{argmax}_{l}\left\{\Delta_{i l}^{\alpha}, \Delta_{l j}^{\alpha}\right\}$ for $\Omega_{\alpha}$, $\alpha \in\{S, R\}$. The graphs for each class were built separately with differences $\Delta_{i j}^{S}$ and $\Delta_{i j}^{R}$ for $\Omega_{S}$ and $\Omega_{R}$ classes respectively.

The process of selection and addition could be continued while the pairs with differences higher than $\varepsilon$ were available. When the process was completed a set of patterns as a graph was obtained. The level of $\varepsilon$ defined the degree of patterns association with sensitivity or resistance to TNF.

\section{Results. Hierarchic patterns}

At the preliminary stage of the method the markers with statistically significant positive correlations with resistance to TNF were defined. The results of calculation are summarized in Table 1.

The first column of the table contains marker descriptions by the form of " $\mathbf{D}<$ number of chromosome $>\mathbf{M}<$ genetic 
distance>". The levels of correlation significance are indicated by P-level in the next column. The third column involves the values of Kendall $\tau$ and Spearman $r_{s}$ rank correlations.

TABLE I

CORRELATIONS BETWEEN MARKERS AND RESISTANCE TO TNF

\begin{tabular}{l|l|l}
\hline \hline \multicolumn{1}{c|}{ Markers } & \multicolumn{1}{|c}{ P-level } & \multicolumn{1}{c}{$\tau ; r_{s}$} \\
\hline D1M236 & $\mathrm{p}=, 00035$ & $+0,22 ;+0,23$ \\
D1M132 & $\mathrm{p}=, 00233$ & $+0,19 ;+0,2$ \\
D1M74 & $\mathrm{p}=, 07947$ & $+0,23 ;+0,25$ \\
D8M6 & $\mathrm{p}=, 04497$ & $+0,08 ;+0,08$ \\
D11M4 & $\mathrm{p}=, 05326$ & $+0,12 ;+0,13$ \\
D11M99 & $\mathrm{p}=, 07845$ & $+0,1 ;+0,1$ \\
D11M333 & $\mathrm{p}=, 01823$ & $+0,1 ;+0,1$ \\
D15M224 & $\mathrm{p}=, 00384$ & $-0,17 ;-0,18$ \\
D15M7 & $\mathrm{p}=, 07413$ & $-0,08 ;-0,08$ \\
D1M236 & $\mathrm{p}=, 00035$ & $+0,22 ;+0,23$ \\
D1M132 & $\mathrm{p}=, 00233$ & $+0,19 ;+0,2$ \\
D1M74 & $\mathrm{p}=, 07947$ & $+0,23 ;+0,25$ \\
D8M6 & $\mathrm{p}=, 04497$ & $+0,08 ;+0,08$ \\
D11M4 & $\mathrm{p}=, 05326$ & $+0,12 ;+0,13$ \\
D15M224 & $\mathrm{p}=, 00384$ & $-0,17 ;-0,18$ \\
D15M7 & $\mathrm{p}=, 07413$ & $-0,08 ;-0,08$ \\
\hline \hline
\end{tabular}

Fig. 1 demonstrates that positive correlation matches to the case when the resistant genotype connects with the resistant phenotype. The values of correlations for marker «M1D132» are $\tau=+0.19, r_{s}=-0.2$.

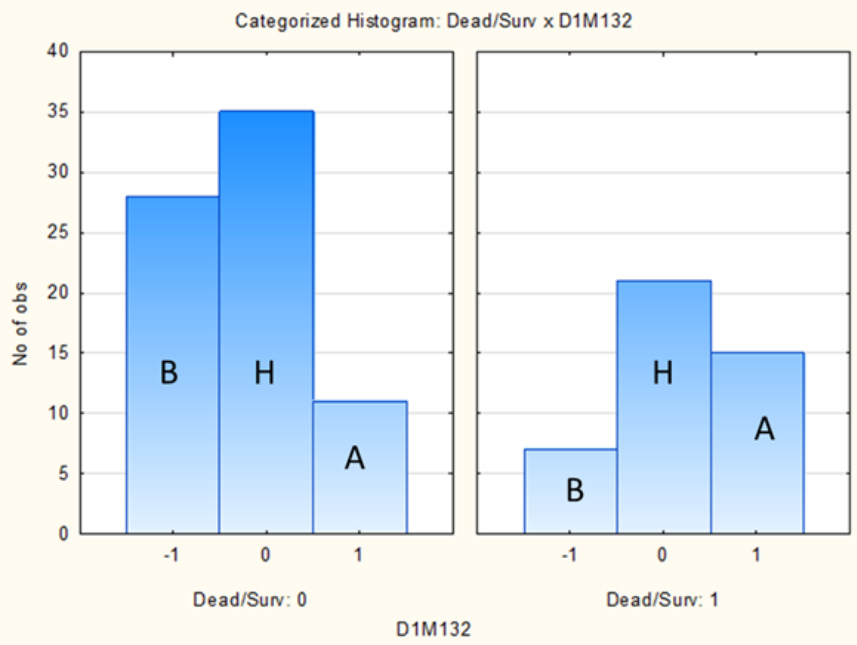

Fig. 1. Categorized histogram for marker «M1D132» by types of phenotype.

The frequency of markers values in Fig.1 show that the genotype «A» prevails in resistant phenotype group (right chart), and the genotype «B» prevails in sensitive phenotype group (left chart).

The opposite case is presented in Fig. 2. The values of correlations for marker «M15D224» are $\tau=-0.17, r_{s}=$ -0.18 .

The charts demonstrate that the genotype «B» prevails in the resistant phenotype group (right chart), and the genotype «A» slightly prevails in the sensitive phenotype group (left chart). This indicates that the negative values of correlation are in contrast to the hypothesis that the resistant genotype connects with the resistant phenotype.

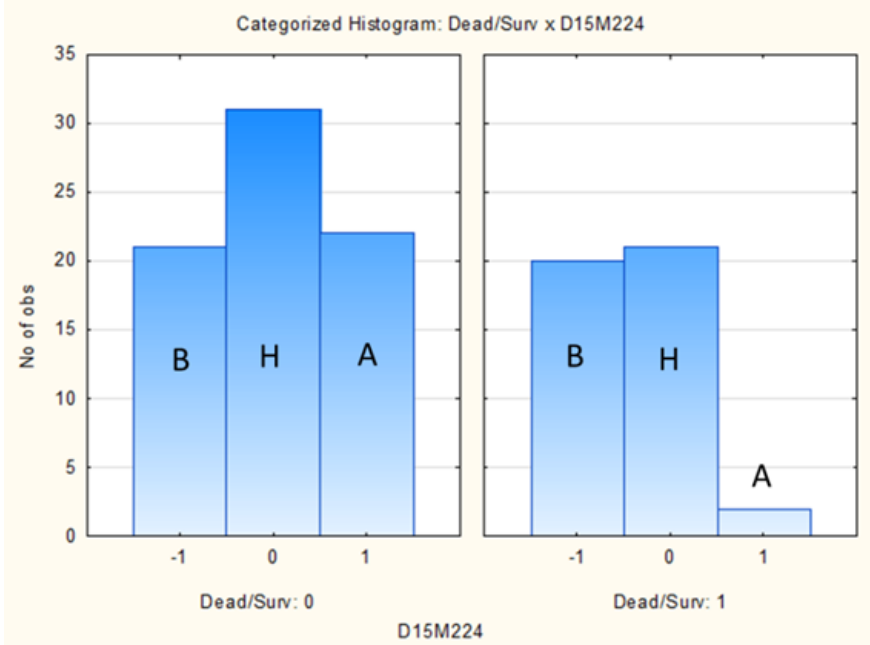

Fig. 2. Categorized histogram for marker M15D224 by types of phenotype.

The hierarchic patterns obtained by the algorithm were presented by the form illustrated in Fig.3.

\begin{tabular}{|c|c|c|c|c|c|c|}
\hline \multirow{2}{*}{\multicolumn{2}{|c|}{ Level 1}} & \multicolumn{2}{|c|}{ Level 2} & \multicolumn{2}{|c|}{ Level 3} & \multirow[b]{2}{*}{12} \\
\hline & & D10M7=H & 0.680 & D3M201=H & 0.706 & \\
\hline $\mathrm{D} 1 \mathrm{M} 236=\mathrm{B}$ & 0.338 & & & & & \\
\hline & & D19M133=H & 0.680 & D3M110 $=\mathrm{H}$ & 0.824 & 14 \\
\hline & & & & D3M211=H & 0.706 & 12 \\
\hline
\end{tabular}

Fig. 3. Hierarchic patterns for sensitive phenotype with value «B» of marker «D1M236» in the root.

The following hierarchic patterns are showed in Fig. 3:

(D1M236=B; D10M7=H; D3M201=H), 12 objects from $\Omega_{S}$ are corresponded to the pattern;

(D1M236=B; D19M133=H; D3M110=H) for 14 objects from $\Omega_{S}$;

(D1M236=B; D19M133=H; D3M211=H) for 12 objects from $\Omega_{S}$.

The hierarchic structure in Figure 3 also contains frequency of combinations:

the frequency of value D1M236=B is 0.338 among the objects $\Omega_{S}$;

the frequency of value $\mathrm{D} 10 \mathrm{M} 7=\mathrm{H}$ is 0.68 among the objects $\Omega_{S}$ with D1M236=B;

the frequency of value D3M201=H is 0.706 among the objects $\Omega_{S}$ with D1M236=B and D10M7=H.

The fragment of 3-levels hierarchic patterns building results are presented in Table 2 . We use $P_{\text {opt }}$ equal 0.4 on $2^{\mathrm{d}}$ and $3^{\text {rd }}$ levels.

Table 2 contains patterns with high number corresponding objects. The results show that the most significant patterns for sensitive phenotype include the markers with " $\mathrm{B}$ " genotype located on $1^{\text {st }}, 8^{\text {th }}$ and $11^{\text {th }}$ chromosomes. The patterns containing markers with negative values of correlation were also built. The patterns similar to $(\mathrm{M} 15 \mathrm{D} 224=\mathrm{A} ; \mathrm{D} 15 \mathrm{M} 7=\mathrm{A}$; D4M196=B) could confirm the contradiction to the hypothesis that the resistant genotype connects with the resistant phenotype for markers with negative values of correlation. 
TABLE II

HIERARCHIC PATTERNS BUILDING RESULTS

\begin{tabular}{|c|c|c|c|c|c|c|}
\hline \multicolumn{2}{|c|}{ Level 1} & \multicolumn{2}{|c|}{ Level 2} & \multicolumn{2}{|c|}{ Level 3} & $\begin{array}{c}\text { Number } \\
\text { of obj. }\end{array}$ \\
\hline \multicolumn{7}{|c|}{ Sensitive phenotype group } \\
\hline \multirow[t]{2}{*}{ D1M373=B } & 0.270 & D1M132=B & 0.676 & D11M294=A & 0.440 & 11 \\
\hline & & D1M458=B & 0.568 & D8M13=B & 0.524 & 11 \\
\hline \multirow[t]{2}{*}{$\mathrm{D} 1 \mathrm{M} 236=\mathrm{B}$} & 0.285 & $\mathrm{D} 1 \mathrm{M} 132=\mathrm{B}$ & 0.795 & $\mathrm{D} 4 \mathrm{M} 196=\mathrm{B}$ & 0.419 & 13 \\
\hline & & $\mathrm{D} 1 \mathrm{M} 458=\mathrm{B}$ & 0.487 & $\mathrm{D} 6 \mathrm{M} 274=\mathrm{A}$ & 0.526 & 10 \\
\hline D1M236=B & 0.285 & D1M458=B & 0.487 & $\mathrm{D} 8 \mathrm{M} 120=\mathrm{B}$ & 0.526 & 10 \\
\hline \multirow[t]{2}{*}{$\mathrm{D} 1 \mathrm{M} 132=\mathrm{B}$} & 0.307 & D1M236=B & 0.738 & D4M196=B & 0.419 & 13 \\
\hline & & D1M373=B & 0.595 & D11M294=A & 0.440 & 11 \\
\hline \multirow[t]{2}{*}{$\mathrm{D} 1 \mathrm{M} 102=\mathrm{B}$} & 0.270 & $\mathrm{D} 1 \mathrm{M} 458=\mathrm{B}$ & 0.622 & $\mathrm{D} 8 \mathrm{M} 6=\mathrm{B}$ & 0.435 & 10 \\
\hline & & $\mathrm{D} 1 \mathrm{M} 236=\mathrm{B}$ & 0.486 & $\mathrm{D} 4 \mathrm{M} 196=\mathrm{B}$ & 0.500 & 9 \\
\hline \multirow[t]{2}{*}{$\mathrm{D} 1 \mathrm{M} 458=\mathrm{B}$} & 0.350 & $\mathrm{D} 1 \mathrm{M} 102=\mathrm{B}$ & 0.479 & D8M6=B & 0.435 & 10 \\
\hline & & $\mathrm{D} 1 \mathrm{M} 373=\mathrm{B}$ & 0.438 & $\mathrm{D} 8 \mathrm{M} 13=\mathrm{B}$ & 0.524 & 11 \\
\hline \multirow[t]{2}{*}{ D11M294=B } & 0.299 & $\mathrm{D} 11 \mathrm{M} 4=\mathrm{B}$ & 0.537 & $\mathrm{D} 10 \mathrm{M} 71=\mathrm{B}$ & 0.455 & 10 \\
\hline & & D11M333=B & 0.415 & D8M6=A & 0.588 & 10 \\
\hline \multirow[t]{2}{*}{$\mathrm{D} 11 \mathrm{M} 4=\mathrm{B}$} & 0.336 & $\mathrm{D} 11 \mathrm{M} 294=\mathrm{B}$ & 0.478 & $\mathrm{D} 10 \mathrm{M} 71=\mathrm{B}$ & 0.455 & 10 \\
\hline & & $\mathrm{D} 8 \mathrm{M} 120=\mathrm{B}$ & 0.413 & D5M394=A & 0.526 & 10 \\
\hline $\mathrm{D} 11 \mathrm{M} 333=\mathrm{B}$ & 0.307 & $\mathrm{D} 11 \mathrm{M} 294=\mathrm{B}$ & 0.405 & $\mathrm{D} 8 \mathrm{M} 6=\mathrm{A}$ & 0.588 & 10 \\
\hline \multirow[t]{3}{*}{$\mathrm{D} 15 \mathrm{M} 224=\mathrm{A}$} & 0.299 & $\mathrm{D} 15 \mathrm{M} 7=\mathrm{A}$ & 0.780 & $\mathrm{D} 4 \mathrm{M} 196=\mathrm{B}$ & 0.438 & 14 \\
\hline & & & & D8M6=B & 0.406 & 13 \\
\hline & & $\mathrm{D} 15 \mathrm{M} 104=\mathrm{A}$ & 0.610 & $\mathrm{D} 4 \mathrm{M} 196=\mathrm{B}$ & 0.440 & 11 \\
\hline \multicolumn{7}{|c|}{ Resistant phenotype group } \\
\hline \multirow[t]{2}{*}{$\mathrm{D} 1 \mathrm{M} 373=\mathrm{A}$} & 0.329 & D1M102=A & 0.560 & D15M224=B & 0.500 & 7 \\
\hline & & $\mathrm{DXM} 234=\mathrm{A}$ & 0.520 & $\mathrm{D} 5 \mathrm{M} 98=\mathrm{B}$ & 0.538 & 7 \\
\hline \multirow[t]{2}{*}{$\mathrm{D} 1 \mathrm{M} 236=\mathrm{A}$} & 0.368 & D5N4=B & 0.536 & $\mathrm{D} 16 \mathrm{M} 189=\mathrm{B}$ & 0.400 & 6 \\
\hline & & DXM234=A & 0.536 & D5M98=B & 0.400 & 6 \\
\hline \multirow[t]{2}{*}{$\mathrm{D} 1 \mathrm{M} 132=\mathrm{A}$} & 0.395 & D5M98=B & 0.567 & $\mathrm{D} 7 \mathrm{M} 246=\mathrm{B}$ & 0.412 & 7 \\
\hline & & & & $\mathrm{D} 8 \mathrm{M} 120=\mathrm{B}$ & 0.412 & 7 \\
\hline \multirow[t]{2}{*}{$\mathrm{D} 1 \mathrm{M} 102=\mathrm{A}$} & 0.355 & $\mathrm{D} 2 \mathrm{M} 340=\mathrm{A}$ & 0.556 & D5M98=B & 0.533 & 8 \\
\hline & & & & D6M274=B & 0.400 & 6 \\
\hline \multirow[t]{2}{*}{$\mathrm{D} 1 \mathrm{M} 458=\mathrm{A}$} & 0.303 & $\mathrm{D} 1 \mathrm{M} 236=\mathrm{A}$ & 0.478 & $\mathrm{D} 15 \mathrm{M} 224=\mathrm{B}$ & 0.545 & 6 \\
\hline & & $\mathrm{D} 2 \mathrm{M} 340=\mathrm{A}$ & 0.478 & D6M274=B & 0.545 & 6 \\
\hline \multirow[t]{2}{*}{ D11M294=A } & 0.289 & $\mathrm{D} 5 \mathrm{M} 98=\mathrm{B}$ & 0.500 & $\mathrm{DXM} 249=\mathrm{A}$ & 0.545 & 6 \\
\hline & & $\mathrm{D} 11 \mathrm{M} 4=\mathrm{A}$ & 0.500 & $\mathrm{D} 3 \mathrm{M} 22=\mathrm{B}$ & 0.545 & 6 \\
\hline D11M4=A & 0.289 & D11M294=A & 0.500 & D3M22=B & 0.545 & 6 \\
\hline D11M99=A & 0.289 & D9M155=A & 0.545 & $\mathrm{D} 5 \mathrm{M} 98=\mathrm{B}$ & 0.500 & 6 \\
\hline D11M333=A & 0.316 & D15M224=B & 0.417 & D1M373=A & 0.600 & 6 \\
\hline
\end{tabular}

The results also show that the most significant patterns for resistant phenotype primarily include the markers with "A" genotype located on $1^{\text {st }}$ and $11^{\text {th }}$ chromosomes. Furthermore the study of resistant phenotype patterns demonstrated the great variety of examined objects genotype.

\section{Results. Patterns as a graph}

As mentioned previously the method is based on frequency calculation of paired occurrence of marker values for each class of objects and on presentation the results as a graph. The part of graphs built on the base of pairs with highest differences $\Delta^{S}$ and $\Delta^{R}$ are presented in Table 3 . The paired frequency $p_{i j}^{S}, p_{i j}^{R}$ were used as the weights of the edges.

Combinations for $\Omega_{S}$ class demonstrate amalgamation of markers with genotype "B". Algorithm combines the markers of $1^{\text {st }}$ and $11^{\text {th }}$ chromosomes into a set of patterns, for example: (D11M99=B; D11M333=B; D11M4=B) or (D1M373=B; D1M236=B; D1M132=B).

The algorithm combines the markers with genotype " $\mathrm{A}$ " for $\Omega_{R} \quad$ class, for example: (D1M373=A; D1M236=A; D1M132=A; D1M102 = A).

The patterns presented in Table 3 can confirm the accordance to the hypothesis that the resistant genotype connects with the resistant phenotype. Developed patterns demonstrate the occurrence of multiple correlations between markers within classes.

The revealed graphs also show that the most significant markers responsible for sensitivity or resistance to TNF are located on $1^{\text {st }}$ and $11^{\text {th }}$ chromosomes. Consequently the accordance of object with patterns for $\Delta^{S}$ and $\Delta^{R}$ classes should increase the level of sensitivity or resistance to TNF respectively.
TABLE Ш

PATTERNS BUILDING RESULTS

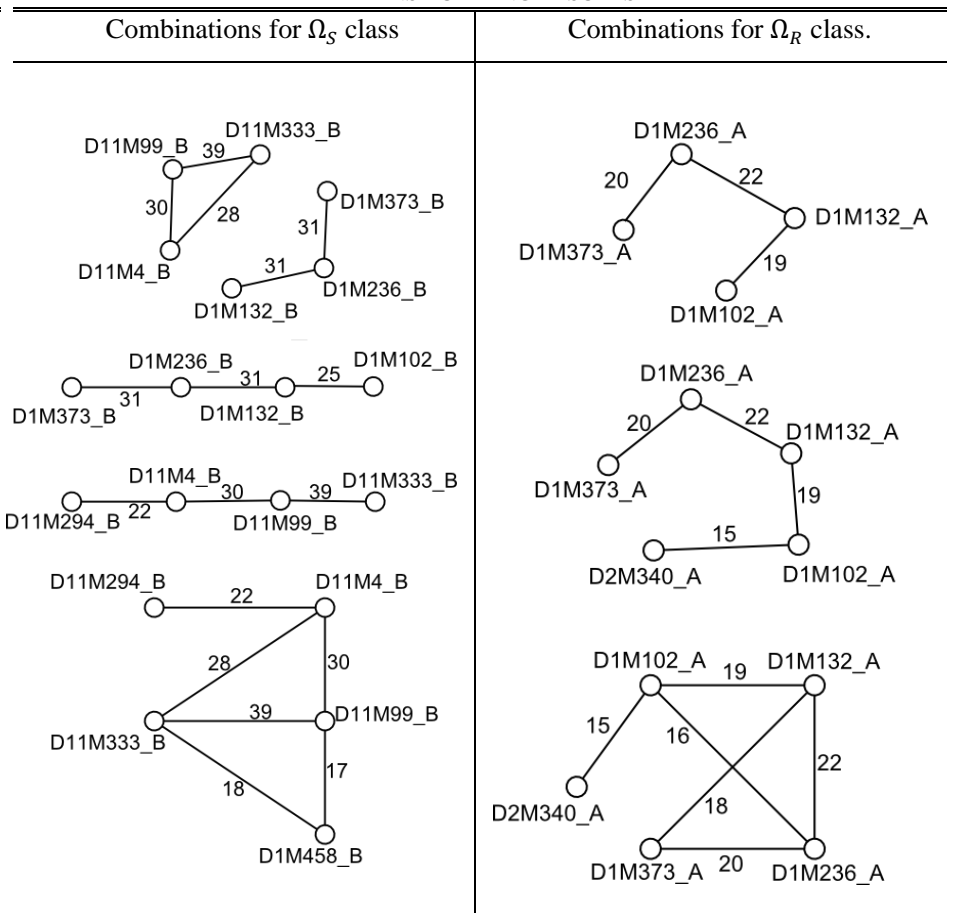

\section{CONCLUSION}

Our investigation was focused on development of methods which would allow us to determine the multiple variables effect on a discrete result. The developed methods were used to find the marker or a group of markers responsible for sensitivity or resistance to TNF. We used two types of patterns building methods in the study. The obtained results reveal some combination of markers which might significantly affect the genotype.

At the first stage the markers with significant positive correlations with resistance to TNF were defined. Such correlations were detected for $1^{\text {st }}, 8^{\text {th }}$ and $11^{\text {th }}$ chromosome markers.

We found that the developed patterns have a great variety. Implementation of the hierarchic patterns building method showed that even patterns with high occurrence frequency matched to a few objects. The results provided evidence of significant correspondence of $1^{\text {st }}$ and $11^{\text {th }}$ chromosome markers with resistance to TNF. The same results were demonstrated by the patterns built according to the graph method.

The study of genetic information demonstrated the great variety of examined objects. The variety essentially complicated the process of finding a group of markers responsible for sensitivity or resistance to TNF.

Further work should therefore include a more detailed study of $1^{\text {st }}$ and $11^{\text {th }}$ chromosome markers and further development of pattern building methods.

\section{ACKNOWLEDGEMENT}

The authors would like to thank the Laboratory of Molecular Genetics of Constitutive Immunity at Petrozavodsk State University for collection and providing initial data. Also 
we would like to express our gratitude to prof. A.N. Poltorak for ideas about methods and some useful recommendations.

\section{REFERENCES}

[1] B. Beutler, A. Poltorak "Sepsis and evolution of the innate immune response". Crit Care Med. 2001; 29(7 Suppl):S2-6; discussion S6-7.

[2] A. Kruglov, A. Tumanov, S. Grivennikov, Y. Shebzukhov, A. Kuchmiy, G. Efimov, M. Drutskaya, J. Scheller, D. Kuprash, S. Nedospasov. "Modalities of experimental TNF blockade in vivo: mouse models". Adv Exp Med Biol. 2011. 691:421-31.

[3] P. Armitage "Statistical Methods in Medical Research". Wiley. 2002.

[4] X.-H. Zhou, N. Obuchowski, D. McClish "Statistical Methods in Diagnostic Medicine". John Wiley \& Sons. 2011.

[5] G. Zheng, Y. Yang, X. Zhu, R. C. Elston "Analysis of Genetic Association Studies". Springer, 2012.
[6] G. Clarke, C. Anderson, K. Zondervan "Basic statistical analysis in genetic case-control studies". Online: http://www.ncbi.nlm.nih.gov/ pmc/articles/PMC3154648/.

[7] S. Lin, H. Zhao "Handbook on Analyzing Human Genetic Data: Computational Approaches and Software". Springer, 2009.

[8] E. Zeggini, A. Morris "Analysis of Complex Disease Association Studies: A Practical Guide". Academic Press, 2010.

[9] StatSoft Electronic Statistics Textbook. Online: http://www.statsoft.com/Textbook/Classification-Trees

[10] J. Han, M. Kamber, J. Pei "Data Mining: Concepts and Techniques". Elsevier. 2011.

[11] S.A. Aivazyn, I.S. Yenyukov, L.D. Meshalkin "Applied Statistic. Study of Relationships". Finansy i statistika. Moscow. 1985. (in Russian).

[12] S.A. Aivazyn, I.S. Yenyukov, L.D. Meshalkin "Bases of modelling and initial data processing". Finansy i statistika. Moscow. 1983.(in Russian). 